\title{
El Instituto Investigación en Ciencia Animal y Tecnologia impulsa la Publicación en la Educación Superior
}

J Selva Andina Anim Sci. 2016; 3(1):1.

El Instituto de Investigación Ciencia Animal y Tecnología de la Carrera de Medicina Veterinaria y Zootecnia viene desarrollando la tarea de investigación y participando de los concursos de los proyectos de investigación para acceder a los recursos de impuesto directo a los hidrocarburos (IDH) desde la gestión 2012, enmarcados en las líneas de investigación consistentes en Reproducción Animal y Biotecnología, Alimentación y Nutrición, Salud animal, Laboratorio y fauna silvestre - Medio Ambiente, tomando como punto de inicio de la investigación en las problemáticas locales, regionales en el rubro de la producción, generando alternativas de solución orientados a mejorar la reproducción y reproductividad que es una de las demandas de la sociedad dedicado al rubro productivo.

El Instituto de Investigación en Ciencia Animal y Tecnología (IICAT) de la Carrera de Medicina Veterinaria y Zootecnia ejecutó el proyecto concursable titulado "Valor Nutricional de la Pradera Nativa y Consumo de Alimento en Llamas en el Altiplano de Bolivia", teniendo como objetivo general Evaluar el valor nutricional de la pradera nativa y consumo de alimento en llamas en el Provincia José Manuel Pando, Municipio de Santiago de Machaca del Altiplano de Bolivia. De los resultados de investigación se generaron dos artículos científicos titulados; Determinación del valor nutricional de la pradera nativa provincia José Manuel Pando, Municipio de Santiago de Machaca y Determinación del consumo de alimento y su calidad nutricia en llamas de la provincia José Manuel Pando, Municipio de Santiago de Machaca.

La metodología de investigación desarrollada se inició desde el lugar de origen de los problemas que se generan en el campo basadas en aquellas que son de prioridad que tengan connotaciones serias que afectan a los productores y a partir de ella son tomadas en cuenta para su estudio en búsqueda de una solución científica. Una vez realizada las investigaciones, los resultados son, procesadas en artículos y publicadas en una Revista Científica, esto se constituyen una fuente de información destinado para docentes, estudiantes, organizaciones de productores, asociaciones y Gobiernos Municipales, como un apoyo de referencia para el sector estudiantil y ganadero.

La integridad de los resultados y la información expuesta en los artículos científicos es de responsabilidad exclusiva de los autores.

Instituto de Investigación en Ciencia Animal y Tecnología (IICAT) Medicina Veterinaria y Zootecnia Universidad Pública de El Alto (UPEA). Km 25, Carretera Internacional La Paz-Desaguadero. La Paz-Bolivia. Dirección de contacto: Tel +591-2-2844177, Fax +591-2-2845800 E-mail: jorge_marsal@yahoo.es (C) 2016. Journal of the Selva Andina Animal Science. Bolivia. Todos los derechos reservados 